\title{
Fluorescence-guided surgery of malignant gliomas based on 5-aminolevulinic acid: paradigm shifts but not a panacea
}

\section{Aliasgar Moiyadi, Parvez Syed and Sanjeeva Srivastava}

We read with interest the article (Fluorescence-guided surgery with live molecular navigation - a new cutting edge. Nature Rev. Cancer 13, 653-662 $(2013))^{1}$ by Quyen T. Nguyen and Roger Y. Tsien and agree with their opinion on fluorescence-guided resection of tumours and the application of intraoperative fluorescence in both diagnosis and therapy across a range of diseases. We would like to underscore the role of live molecular navigation (LMN) in the resection of glioblastomas. Safe maximal resection is crucial in brain tumours, where even the slightest damage to normal brain tissue can result in debilitating morbidity. Fluorescence-guided surgery (FGS) of malignant gliomas based on 5-aminolevulinic acid (ALA) has been useful in determining the borders of tumours, thereby making the radical resection of tumours easier ${ }^{2}$. As Nguyen and Tsien acknowledge $^{1}$, malignant gliomas are probably the only clinical application for which FGS has been examined using a randomized controlled clinical trial ${ }^{3}$. FGS is now accepted as a standard of care in many different parts of the world and has resulted in a paradigm shift, encouraging neurosurgeons to resect radically.
Although this sounds exciting, there exist real limitations, which need to be considered before ascribing unequivocal benefits to FGS. This technique is useful in "contrastenhancing, potentially resectable gliomas" (REF. 3); however, it is not a panacea. The major limitation of ALA-based FGS in glioma resection is its inconsistency ${ }^{2}$. Although strong fluorescence can be specific for tumour tissue and have a high positive predictive value, ALA-based FGS has a less-than-desirable sensitivity and a negative predictive value: it is unlikely to lead to overdiagnosis, but it is likely to miss areas where coalescent, highly cellular tumour tissue might be present. This is a substantial concern if $\mathrm{LMN}$ is to be reliably used for guiding the extent of resection. The variability of fluorescence in malignant gliomas could be a consequence of variable uptake kinetics that are related to blood-brain barrier dynamics and cell transport mechanisms, or it could be a consequence of the variable metabolic potential of the intracellular machinery, and each of these in turn could be a surrogate marker of the tumour biology $y^{4-6}$; this needs further investigation.

Further, it must be remembered that intraoperative visualization of fluorescence depends on the sensitivity of both the microscope filters and the camera used.
Indeed, using ultrasensitive instruments such as confocal microscopy, even very low quanta of fluorescence can be detected ${ }^{7,8}$, but this technique is still unavailable for routine clinical use. Undoubtedly, further research will lead to improvements in this field of surgery and extend the applications in LMN.

Aliasgar Moiyadi is at the Department of Neurosurgery, Advanced Center for Treatment Research and Education in Cancer, Tata Memorial Center, Kharghar, Navi Mumbai 410210, India.

Parvez Syed and Sanjeeva Srivastava are at the Wadhwani Research Center for Biosciences and Bioengineering, Department of Biosciences and Bioengineering, Indian Institute of Technology Bombay, Powai, Mumbai 400 076, India

Correspondence to S.S e-mail: sanjeeva@iitb.ac.in doi: 10.1038/nrc3566-c1

1. Nguyen, Q. T. \& Tsien, R. Y. Fluorescence-guided surgery with live molecular navigation - a new cutting edge. Nature Rev. Cancer 13, 653-662 (2013).

2. Stummer, W. et al. Fluorescence-guided resection of glioblastoma multiforme by using 5 -aminolevulinic acid-induced porphyrins: a prospective study in 52 consecutive patients. J. Neurosurg. 93, 1003-1013 (2000).

3. Stummer, W. et al. Fluorescence-guided surgery with 5-aminolevulinic acid for resection of malignant glioma: a randomised controlled multicentre phase III trial. Lancet Oncol. 7, 392-401 (2006).

4. Colditz, M. J., Leyen, K. \& Jeffree, R. L. Aminolevulinic acid (ALA)-protoporphyrin IX fluorescence guided tumour resection. Part 2: theoretical, biochemical and practical aspects. J. Clin. Neurosci. 19, 1611-1616 (2012).

5. Rodriguez, L. et al. Study of the mechanisms of uptake of 5-aminolevulinic acid derivatives by PEPT1 and PEPT2 transporters as a tool to improve photodynamic therapy of tumours. Int. J. Biochem. Cell Biol. 38, 1530-1539 (2006).

6. Xiang, J. et al. PEPT2-mediated transport of 5 -aminolevulinic acid and carnosine in astrocytes. Brain Res. 1122, 18-23 (2006).

7. Sanai, N. et al. Intraoperative confocal microscopy in the visualization of 5-aminolevulinic acid fluorescence in lowgrade gliomas. J. Neurosurg. 115, 740-748 (2011).

8. Nabavi, A. et al. Five-aminolevulinic acid for fluorescence-guided resection of recurrent malignant gliomas: a phase II study. Neurosurgery 65 , 1070-1076 (2009)

Competing interests statement

The authors declare no competing interests. 